\title{
HST/FOS time-resolved spectral mapping of IP Pegasi at the end of an outburst ${ }^{\star}$
}

\author{
R. K. Saito ${ }^{1}$, R. Baptista ${ }^{1}$, and K. Horne ${ }^{2}$ \\ ${ }^{1}$ Departamento de Física, Universidade Federal de Santa Catarina, Trindade, 88040-900, Florianópolis, SC, Brazil \\ e-mail: saito@astro.ufsc.br \\ 2 School of Physics and Astronomy, University of St. Andrews, KY16 9SS, Scotland, UK
}

Received 5 October 2004 / Accepted 21 December 2004

\begin{abstract}
We report an eclipse mapping analysis of time-resolved ultraviolet spectroscopy covering three eclipses of the dwarf nova IP Pegasi on the late decline of the 1993 May outburst. The eclipse maps of the first run show evidence of one spiral arm, suggesting that spiral structures may still be present in the accretion disc 9 days after the onset of the outburst. In the spatially resolved spectra the most prominent lines appear in emission at any radius, being stronger in the inner disc regions. The spectrum of the gas stream is clearly distinct from the disc spectrum in the intermediate and outer disc regions, suggesting the occurrence of gas stream overflow. The full width half maximum of C IV is approximately constant with radius, in contrast to the expected $v \propto R^{-1 / 2}$ law for a gas in Keplerian orbits. This line probably originates in a vertically extended region (chromosphere + disc wind). The uneclipsed component contributes $\sim 4 \%$ of the flux in C IV in the first run, and becomes negligible in the remaining runs. We fit stellar atmosphere models to the spatially resolved spectra. The radial run of the disc color temperature for the three runs is flatter than the expected $T \propto R^{-3 / 4}$ law for steady-state optically thick discs models, with $T \simeq 20000 \mathrm{~K}$ in the inner regions and $T \simeq 9000 \mathrm{~K}$ in the outer disc regions. The solid angles that result from the fits are smaller than expected from the parameters of the system. The radial run of the solid angle suggests that the disc is flared in outburst, and decreases in thickness toward the end of the outburst.
\end{abstract}

Key words. stars: individual: IP Peg - stars: binaries: eclipsing - methods: data analysis

\section{Introduction}

Accretion discs are cosmic devices that allow matter to efficiently accrete over a compact source by removing its angular momentum via viscous stresses while transforming gravitational potential energy into head and, thereafter, radiation (Frank et al. 1992). Dwarf novae are excellent environments for advances in the knowledge of accretion physics. In these close binaries mass is fed to a white dwarf (the primary) by a Roche lobe filling companion star (the secondary) via an accretion disc which usually dominates the ultraviolet and optical light of the system (Warner 1995).

The observation of accretion discs in dwarf novae is well beyond the capabilities of current direct-imaging techniques as it requires resolving structures on angular scales of microarcseconds. However, the binary nature of these systems allows the application of powerful indirect-imaging techniques such as eclipse mapping (Horne 1985) and Doppler tomography (Marsh \& Horne 1988) to probe the dynamics, structure and the time evolution of their accretion discs (e.g. Baptista 2001).

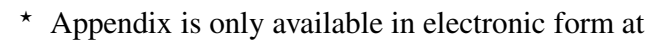
http://www . edpsciences . org
IP Pegasi is an intensively studied deeply eclipsing dwarf nova $\left(P_{\text {orb }}=3.8 \mathrm{~h}\right)$ which shows $1-2$ weeks-long, $\simeq 2 \mathrm{mag}$ outbursts every 60-120 days. In the last decade, several studies, many of them based on indirect-imaging techniques, revealed interesting results about the structure and behavior of the IP Peg accretion disc. Bobinger et al. (1997) performed optical eclipse mapping of IP Peg on decline from an outburst to find that the temperature distribution was much flatter than expected for a steady-state disc, with brightness temperatures in the range $T \simeq 9000-5000 \mathrm{~K}$ for the inner and outer disc regions, respectively.

The discovery of spiral arms in the accretion disc of IP Pegasi during outbursts with Doppler tomography (Steeghs et al. 1997) confirmed the results of hydrodynamical simulations (Stehle 1999). The spiral arms appear because of shocks caused by the tidal effect of the secondary star on the outer parts of the extended accretion disc during outbursts. Steeghs et al. (1997) and Morales-Rueda et al. (2000) confirmed that the spiral structure was a stable feature of IP Peg during outbursts, and that it was still present 5-7 days after the onset of the outburst. Baptista et al. (2000) and Baptista et al. (2002) (hereafter BHS and BHT, respectively) confirmed the presence and recovered the spatial location of spiral arms on the outburst decline from optical eclipse mapping. 
Table 1. Journal of the observations.

\begin{tabular}{ccccc}
\hline \hline Date & UT & & \\
Run & $(1993$ May $)$ & $($ Start $)$ & Eclipse & Phase range \\
\hline IP4 & 27 & $09: 05$ & 22246 & $+0.050,+0.212$ \\
IP5 & 27 & $20: 20$ & 22249 & $+0.011,+0.174$ \\
IP6 & 28 & $07: 34$ & 22252 & $-0.028,+0.136$ \\
IP7 & 30 & $01: 20$ & 22263 & $-0.028,+0.136$ \\
\hline
\end{tabular}

Here we report the results of a time-resolved ultraviolet (UV) spectral mapping experiment of IP Pegasi 8-11 days after the onset of the May 1993 outburst, when the star's optical brightness was almost back to its quiescent level. The analysis is based on observations secured with the Faint Object Spectrograph onboard the Hubble Space Telescope. The observations and the data reduction are described in Sect. 2. The details of the data analysis are given in Sect. 3. In Sect. 4 we investigate the disc spatial structure in the lines and in the continuum and we present spatially resolved spectra of the disc, gas stream and the spectrum of the uneclipsed light. We fit stellar atmosphere models to the disc spectra in order to derive the radial run of the temperature and solid angle of each disc surface element. The results are summarized in Sect. 5. In the Appendix we address the reliability of eclipse mapping reconstructions with light curves of incomplete phase coverage.

\section{Observations}

IP Peg went in outburst on 1993 May 18. The Faint Object Spectrograph (FOS) onboard the Hubble Space Telescope was used to secure time-resolved spectroscopy covering 4 eclipses in 1993 May 27-30, during the late decline from outburst. Figure 1 shows the historical optical light curve of IP Peg around the 1993 May outburst. The times of the HST runs are indicated by vertical dotted lines. The observations of BHT were performed roughly one day before the start of the HST runs and are also indicated in Fig. 1. Therefore, the HST observations allow us the opportunity to track the changes in the disc of IP Peg in the days following the observations reported by BHT.

The HST observations, detailed in Table 1, were performed in the "rapid readout" mode at a time resolution of $5.5 \mathrm{~s}$. To increase the nominal spectral resolution of the FOS spectra, during the acquisition the spectrum was shifted electronically by a quarter of a diode along the 516-diode Digicon array ("1/4 sub-stepping") resulting in a 2064-pixel spectrum with a net exposure time of $1.185 \mathrm{~s}_{\text {pixel }}{ }^{-1}$. The runs were performed with the $4.3 \times 4.3 \operatorname{arcsec}^{2}$ aperture and the G160L grating $\left(\lambda 1100-2500 \AA, \Delta \lambda=1.7 \AA\right.$ pixel $\left.^{-1}\right)$. Because of the relatively large slit aperture, these spectra are contaminated by geo-coronal Ly $\alpha$ emission. The choice of the slit aperture was dictated by the large point-spread function of HST at that epoch (these are pre-COSTAR observations). The runs cover the eclipse cycles 22246 (IP4), 22249 (IP5), 22252 (IP6) and 22263 (IP7), according to the linear ephemeris of Wolf et al. (1993).

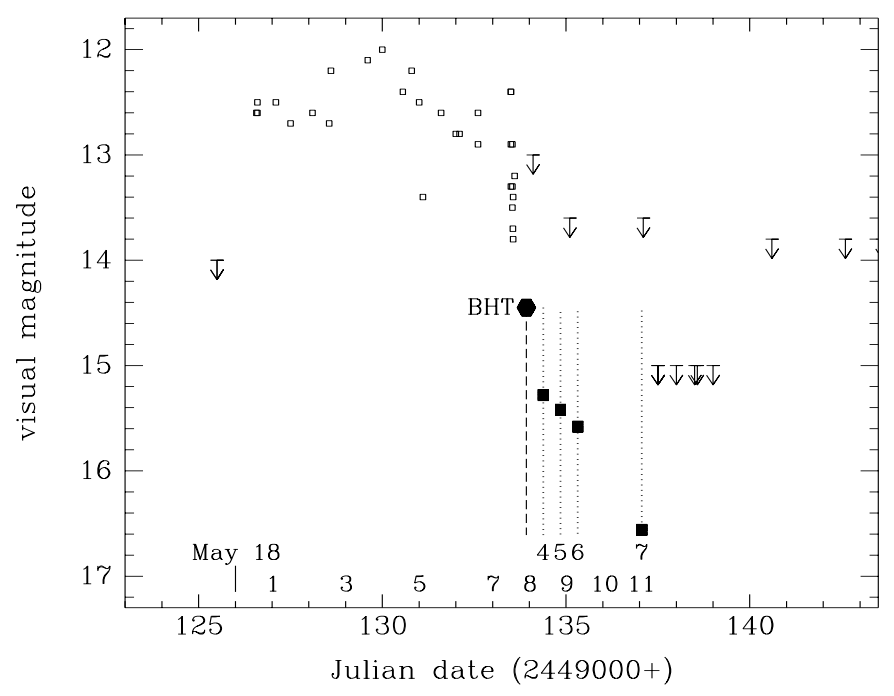

Fig. 1. Visual light curve of IP Peg for the epoch 1993 May-June, constructed from observations made by the AAVSO (open squares). Arrows indicate upper limits on the visual magnitude. The synthetic $V$-band out-of-eclipse magnitude from the data of BHT is shown as a filled hexagon. A vertical dashed line marks the epoch of their observations. Vertical dotted lines mark the epoch of our observations. $A B_{79}$ magnitudes in the range 1100-2500 $\AA$ are shown as filled squares for illustration purposes (labeled runs 4, 5, 6 and 7 (IP+)).

Due to problems with the HST scheduling, the first run (IP4) missed the eclipse. Since that data set contains only the late egress of the light curve it will not be included in the following analysis. Unfortunately, because of the same scheduling problem, the other runs are also miscentered and do not cover the ingress of the eclipse (see Fig. 3).

The observations were reduced with a procedure similar to the standard STSDAS pipeline and included flat-field and geomagnetically induced motion ("GIMP") corrections, background and scattered light substraction, wavelength and absolute flux calibrations.

The average out-of-eclipse UV spectra of runs IP5, IP6 and IP7 are shown in Fig. 2. They show emission lines of Si II $\lambda 1194$, Ly $\alpha$ $\lambda 1216$ (mostly geo-coronal), N V $\lambda 1240,1243$, Si II $\lambda 1300$, C II $\lambda 1336$, Si IV $\lambda 1394,1403$, C IV $\lambda 1549,1551$, HeII $\lambda 1640$, Al II $\lambda 1723$, Al III $\lambda 1859$, Si III $\lambda 1892$, C III $\lambda 2297$, as well as broad absorption bands possibly due to Fe II (e.g. see Fig. 7). The strong and broad emission feature centered at $\simeq 1210 \AA$ is due to geo-coronal Ly $\alpha$ emission filling all the relatively wide slit aperture. Narrow Si II, Ly $\alpha$ and N V line emission from IP Peg are seen on top of the broad geo-coronal component. The position of the second-order Ly $\alpha$ emission is also illustrated in Fig. 2. The broad hump seen in the spectrum at this wavelength (particularly in IP7) suggests a non-negligible contamination by geo-coronal $\operatorname{Ly} \alpha$ at these wavelengths too.

The continuum emission decreases by a factor of 3 from run IP5 to run IP7. The decrease in line strength is more pronounced, with most of the lines roughly disappearing in the continuum at IP7. 


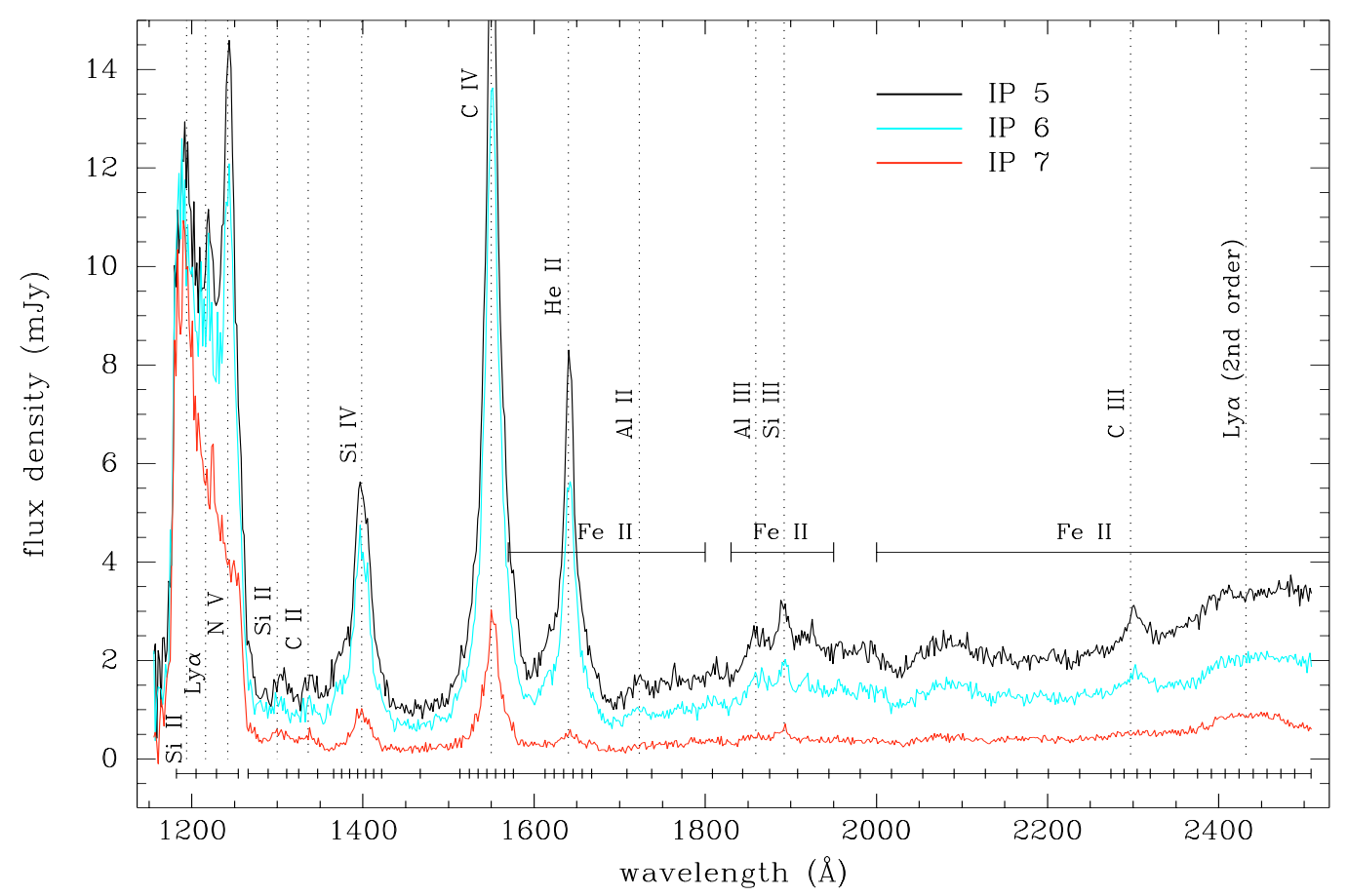

Fig. 2. Average out-of-eclipse spectra of IP Peg during the decline of the 1993 May outburst (phase range +0.10 to +0.14 cycle). The observations correspond to the eclipse cycles 22249 (IP5), 22252 (IP6) and 22263 (IP7), according to the ephemeris of Wolf et al. (1993). Vertical dotted lines mark the emission lines. Horizontal lines mark the Fe II absorption bands. Horizontal ticks indicate the 58 narrows passbands used to extract light curves.

\section{Data analysis}

\subsection{Light-curve construction}

The spectra were divided into a set of 58 narrow passbands, with 23 continuum passbands $16-50 \AA$ wide and 35 passbands for 13 lines (Fig. 2). The strongest emission lines were separated in velocity-resolved bins $2000 \mathrm{~km} \mathrm{~s}^{-1}$ wide, with a bin centered in the rest wavelength $\left(v=0 \mathrm{~km} \mathrm{~s}^{-1}\right)$. For the fainter lines we defined a single bin of $5000-6000 \mathrm{~km} \mathrm{~s}^{-1}$ centered in the rest wavelength. The systemic velocity of IP Peg $\left(\gamma \simeq 30 \mathrm{~km} \mathrm{~s}^{-1}\right)$ (Martin et al. 1989) is both rather uncertain and much smaller than the width of the passbands and was neglected. The $\mathrm{S} / \mathrm{N}$ of the spectra decreases towards shorter wavelengths, which prevented us from extracting a usable light curve blueward of the $\lambda 1194$ line.

For those passbands including emission lines the light curve comprises the total flux at the corresponding bin, with no substraction of a possible continuum contribution.

Light curves were extracted for each passband by computing the average flux on the corresponding wavelength range and phase-folding the results according to the linear ephemeris of Wolf et al. (1993),

$$
T_{\text {mid }}(\mathrm{HJD})=2445615.4156+0.15820616 E,
$$

where $T_{\text {mid }}$ gives the superior conjunction of the white dwarf. The error bars are taken as the standard derivation with respect to the average flux at each phase bin.

IP Peg shows long-term $(\simeq 5 \mathrm{yr})$ cyclical orbital period changes, with departures of the observed mid-eclipse timings of up to 2 min with respect to the ephemeris of Eq. (1).
Fortunately, the HST outburst observations were bracketed by two observations of IP Peg in quiescence in which the egress time of the white dwarf can be easily measured (Baptista et al. 1994). From the contemporary quiescent data we inferred a white dwarf mid-eclipse phase of $\phi_{0}=-0.0082$ cycle for this epoch (adopting an eclipse width of $\Delta \phi=0.0863$ cycle, Wood $\&$ Crawford 1986) and corrected the data accordingly to make the centre of the white dwarf eclipse coincident with phase zero.

Light curves at a selected continuum passband and for the CIV line centre passband are shown in Fig. 3. The incomplete eclipse phase coverage of the runs is clear. Light curves for the IP4 run are also shown. The eclipse in the IP4 continuum is perceptibly wider than in the corresponding C IV light curve and the egress shoulder in the continuum eclipse shape indicates the reappearance of an asymmetric bright structure, possibly the "red" spiral arm (see Sect. 4.1).

\subsection{Eclipse mapping}

Maximum-entropy eclipse mapping techniques (Horne 1985; Baptista \& Steiner 1993) were used to solve for a map of the disc brightness distribution and for the flux of an additional uneclipsed component in each band. The reader is referred to Baptista (2001) for a recent review on the eclipse mapping method.

As our eclipse map we adopted a flat grid of $51 \times 51$ pixels centered on the primary star with side $2 R_{L 1}$, where $R_{L 1}$ is the distance from the disc centre to the inner Lagrangian point. The eclipse geometry is defined by the inclination $i$ and the mass 


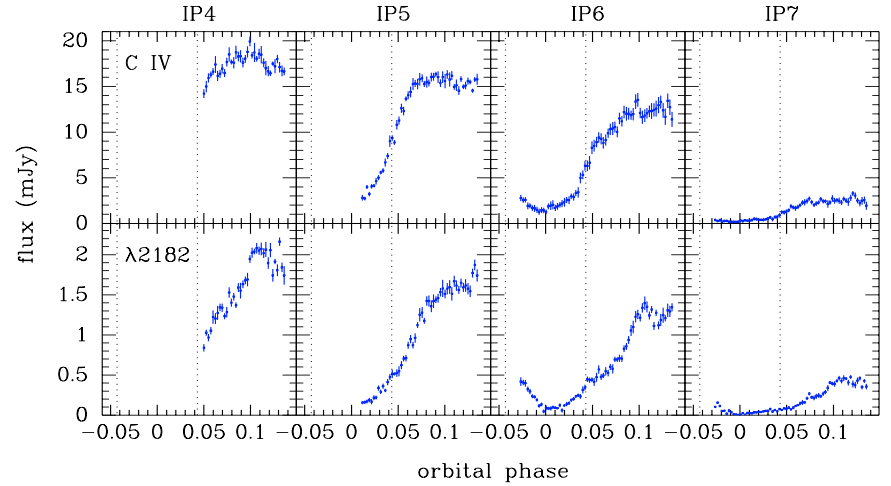

Fig. 3. Light curves for the C IV emission line centre and for a selected continuum passband. Vertical dotted lines mark the ingress/egress phases of the white dwarf.

ratio $q$. The mass ratio $q$ defines the shape and the relative size of the Roche lobes. The inclination $i$ determines the shape and extension of the shadow of the secondary star as projected onto the orbital plane. In this paper we adopted the values of Wood \& Crawford (1986), $q=0.5$ and $i=81^{\circ}$, which correspond to an eclipse phase width of $\Delta \phi=0.0863$ cycle. This combination of parameters ensures that the white dwarf is at the centre of the map. BHT compared eclipse maps obtained with the above geometry with maps derived with the geometry of Marsh (1988) $\left(q=0.58, i=79.5^{\circ}\right)$ and found no perceptible differences in the eclipse maps.

For the reconstructions we adopted the default of limited azimuthal smearing of Rutten et al. (1992), which is better suited for recovering asymmetric structures than the original default of full azimuthal smearing (cf. Baptista et al. 1996). The reader is referred to BHS and Baptista (2001) for eclipse mapping simulations with asymmetric sources which show how the presence of spiral structures affect the shape of the eclipse light curve, and which evaluate the ability of the eclipse mapping method to reconstruct asymmetric structures in eclipse maps. The performance of the eclipse mapping technique for the case of light curves with the incomplete phase coverage of our data sets is discussed in the Appendix A.

The statistical uncertainties in the eclipse maps were estimated with a Monte Carlo procedure (e.g. Rutten et al. 1992). For a given narrow-band light curve a set of 20 artificial light curves is generated, in which the data points are independently and randomly varied according to a Gaussian distribution with standard deviation equals to the uncertainty at that point. The light curves are fitted with the eclipse mapping algorithm to produce a set of randomized eclipse maps. These are combined to produce an average map and a map of the residuals with respect to the average, which yields the statistical uncertainty at each pixel. The uncertainties obtained with this procedure are used to estimate the errors in the derived radial intensity and temperature distributions as well as in the spatially-resolved spectra.

We shall remark that this procedure only accounts for the statistical uncertainty in the eclipse maps. Because each outburst stage is represented by a single light curve, it was not possible to reduce the influence of flickering in the eclipse shape by the usual averaging of many individual light curves. Therefore, the eclipse shapes may be affected by flickering. This could introduce artifacts in the eclipse maps, which are not accounted for by the above statistical analysis. Nevertheless, an inspection of the light curves of Fig. 3 shows that the out-of-eclipse flickering is of relatively low amplitude ( $\sim 10$ per cent) and is further reduced during the eclipse. Moreover, the time scale of individual flares is much shorter than the time scale of the changes in the eclipse shape. Since the eclipse mapping modeling is defined by the overall eclipse shape (see Figs. 4 and 5) one should not expect the fast, low amplitude flickering in the light curve to significantly affect the results.

Light curves and respective eclipse maps at selected passbands for the continuum and for the strongest emission lines are shown in Figs. 4 and 5.

\section{Results}

\subsection{Disc structure}

Maps of the disc surface brightness, calculated by the maximum entropy eclipse mapping method, allow us to obtain spatially resolved information about the disc emission, as well as to probe the spectral and temporal evolution of the accretion disc. In this section we discuss the structures in the eclipse maps of the strongest lines in the spectrum, as well as in selected continuum passbands, and we compare our results with those of previous studies.

Previous optical studies using eclipse mapping methods (BHS; BHT) showed evidences for the presence of spiral arms, which are presumably the consequence of shocks caused by tidal effect of the secondary star in the extended accretion disc during outbursts. These asymmetric arcs were seen as structures $\sim 90^{\circ}$ in azimuth extending from the intermediate to the outer disc regions $\left(R=(0.2-0.6) R_{L 1}\right)$. BHT detected spiral arms during the 1993 May outburst, at the day before the start of our observations. Because our data frame the very last stages of this outburst, they open the perspective to check how long after the start of an outburst are the spiral structures still present in the disc of IP Peg.

Figure 4 shows the light curves and respective eclipse maps for three selected continuum passbands. The IP6 e IP7 light curves show asymmetries at egress phases, indicating the presence of more than one emission source or significant intensity variations along the disc radius. The corresponding eclipse maps show asymmetric structures in the side of the disc that is moving away from the secondary star (the upper hemisphere of the eclipse maps in Fig. 4) which can be associated to the gas stream emission. If this assumptions is right, the fact that the emission traces the ballistic stream trajectory beyond the bright spot position is an evidence of gas stream overflow in IP Peg at this late outburst stage. The azimuthal extension of the asymmetric structure at the disc outer regions in IP6 e IP7 shows that part of the energy in the collision of the stream with the disc edge is dissipated along the disc rim, in the direction of the gas rotation. It is interesting to note that while the outburst reaches its final stages, the continuum maps are progressively dominated by emission along the gas stream region. 


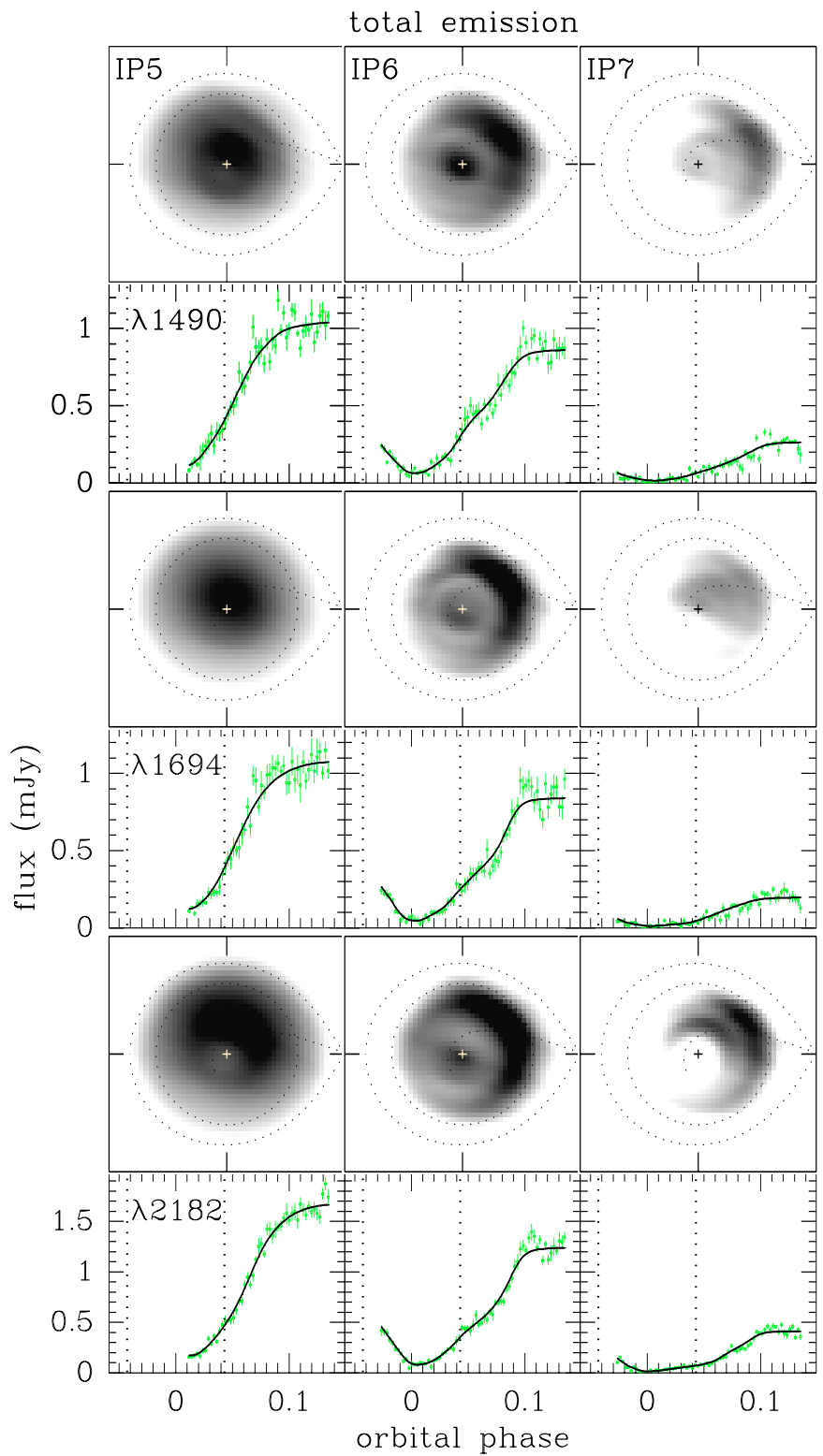

Fig. 4. Data and model light curves and eclipse maps for three selected continuum passbands. The upper panels of each slide show the eclipse maps corresponding to the light curves of the lower panels for the three runs in a logarithmic greyscale. Brighter regions are indicated in black; fainter regions in white. A cross marks the center of the disk; dotted lines show the Roche lobe, the gas stream trajectory, and a disc of radius $0.6 R_{L 1}$; the secondary is to the right of each map and the stars rotate counter-clockwise. The lower panels of each slide show the data (dots with error bars) and model (solid lines) light curves. Vertical dotted lines mark the ingress/egress phases of the white dwarf.

The maximum derivative of the IP5 continuum light curves is displaced towards later phases with respect to white dwarf egress, indicating that the maximum of the brightness distribution does not coincide with the disc centre. Accordingly, the IP5 continuum maps show an arc-shaped asymmetric structure in the disc side moving away from the secondary star with azimuthal width $\sim 90^{\circ}$ and spanning a range of radii $R=$ $(0.2-0.4) R_{L 1}$. The morphology, location, azimuthal and radial width of this arc-shaped structure are similar to those of the

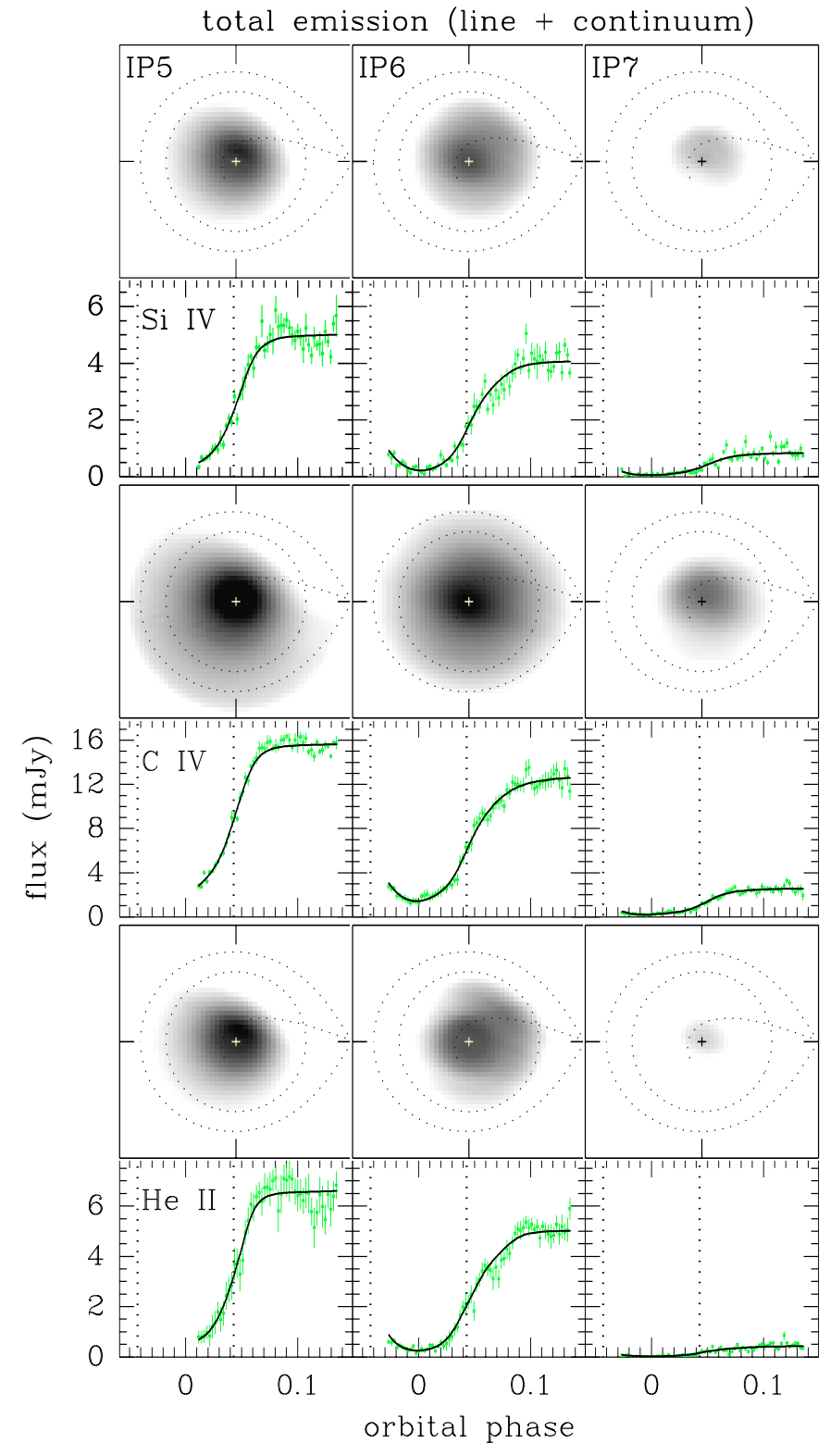

Fig. 5. The upper panels of the each slide show the eclipse maps for the passband centered in the Si IV, C IV and He II emission lines ( $v=$ $0 \mathrm{~km} \mathrm{~s}^{-1}, \Delta v= \pm 1000 \mathrm{~km} \mathrm{~s}^{-1}$ ). The lower panels show the data (dots with error bars) and corresponding model (solid lines) light curves. The notation is similar to that of Fig. 4.

"red" spiral arm seen in BHS. The reader is referred to the Appendix for simulations with and for a discussion of eclipse mapping reconstructions of spiral structures from incomplete phase light curves.

Our eclipse maps do not show evidence of the other ("blue") spiral arm. In analogy with the results of BHS, the blue spiral arm should be located in the region of the eclipse map that is not sampled by the shadow of the secondary star because of the incomplete phase coverage of the IP5 data set (see Fig. A.2 in Appendix A). Since it is not possible to recover the brightness distribution of disc regions for which there is no information in the shape of the light curve (e.g. Baptista 2001), 
it is not surprizing that there is no evidence of the "blue" spiral arm in these maps.

Morales-Rueda et al. (2000) and Steeghs et al. (1997) found evidence of spiral structures in Doppler tomograms of IP Peg, respectively 5-6 and 7 days after the onset of an outburst. Spiral structures were also clearly seen in the eclipse maps of BHT, obtained 8 days after the onset of this same outburst. Our IP5 continuum maps extend the previous results, showing evidence that one spiral arm was still present in the accretion disc of IP Peg 9 days after the start of the outburst.

Figure 5 shows the light curves and respective eclipse maps for the line centre of the three strongest emission lines, Si IV, $\mathrm{C} \mathrm{IV}$ and He II. The velocity-resolved passbands have widths of $\Delta v=2000 \mathrm{~km} \mathrm{~s}^{-1}$ (from -1000 to $+1000 \mathrm{~km} \mathrm{~s}^{-1}$, centered in $v=0 \mathrm{~km} \mathrm{~s}^{-1}$ ). The phase of maximum derivative in the light curves for all runs coincides with the white dwarf egress phase, indicating that the maxima of the distributions coincide with the disc centre. The light curves show deep eclipses with smooth egresses. As a consequence, the resulting maps show broad brightness distributions centered at the white dwarf position with no asymmetric structure that could signal the presence of spiral arms. This is in contrast with the results of BHS, the He I line maps of which show the same asymmetric structures observed in the continuum maps. The strong UV lines are probably produced in a vertically-extended, opaque chromosphere + disc wind, which may veil the underlying emission from the accretion disc and spiral arms.

The eclipse egress occurs perceptibly later in IP6 than in IP5. The small asymmetry observed in the eclipse egress in the IP6 light curves produces the emission in the bright spot region in the corresponding eclipse maps. We note that the line maps contain the line emission plus any possible underlying continuum. Thus, the presence of asymmetric structures in the continuum maps and its absence in the line maps, in addition to the observed spectrum of the uneclipsed component (see Sect. 4.4), is a indication of the vertical extension and large optical depth of the gas from which the lines originate. Our interpretation is that this opaque and vertically extended gas hides the structures observed in the continuum, leading to smooth line maps in which the emission along the bright spot region is much less visible than in the continuum.

The time sequence of eclipse maps show the progressive fading of both line and continuum emission as the outburst ends. The intensity of the maps decrease significantly from run IP6 to IP7. This is particularly true for the Si IV and He II line maps, for which the line emission becomes restricted to the central parts of the disc. In contrast with the smooth, symmetric line maps, the continuum emission at the end of the outburst arises mainly at the bright spot position and along the ballistic stream trajectory.

\subsection{Spatially resolved spectra}

The set of monochromatic eclipse maps allow us to obtain spatially resolved spectra for the accretion disc. In order to separate the disc spectrum at different distances from the disc

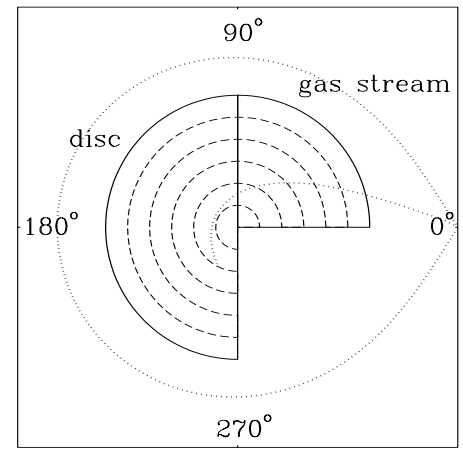

Fig. 6. Diagram showing the regions defined as "disc" and "gas stream". The dashed lines mark the annular regions of width $0.1 R_{L 1}$ used to extract spatially resolved spectra. Dotted lines show the projection of the primary Roche lobe onto the orbital plane and the gas stream trajectory. Azimuths are measured with respect to the line joining both stars and increase counter-clockwise. Four reference azimuths are labeled in the figure.

centre, we divided the disc in concentric annular sections of width $\Delta R=0.1 R_{L 1}$.

Motivated by the distinct emission observed in the bright spot and gas stream region, we separated the eclipse map in two distinct azimuthal regions: "disc" and "gas stream". We define the "gas stream" as the map section between $0^{\circ}$ and $90^{\circ}$, and the "disc" as the region between azimuths $90^{\circ}$ and $270^{\circ}$ (see Fig. 6). We do not include the section between $270^{\circ}$ and $360^{\circ}$ in the "disc" region because there is evidence of contamination by emission from the bright spot in this region (because of the intrinsic azimuthal smearing effect of the eclipse mapping technique) and also because there is evidence that the disc has a non-negligible opening angle (see Sect. 4.5) and this could introduce artifacts in the computation of the average flux of each annulus.

Each point in the spectrum is obtained by averaging the intensity of all pixels inside the corresponding annulus. The statistical uncertainties affecting the average intensities are estimated with the Monte Carlo procedure described in Sect. 3.2.

The spatially resolved disc spectra for the three runs are shown in Fig. 7. The spectra display strong lines of Si IV, C IV and He II that appear in emission at all disc radii. This behavior is similar to that observed for the $\mathrm{H} \alpha$ and $\mathrm{He}$ I lines in the contemporary optical spectral mapping experiment of BHS. We note again the radial extension of the C IV emission: in the disc inner regions the Si IV, C IV and He II lines have comparable strengths but the Si IV and He II lines decrease quickly in intensity with increasing disc radius while the intensity of the CIV line remains approximately at the same level of the inner disc regions. The He II emission is concentrated in the disc centre and is possibly associated to photoionization by radiation from the boundary layer (Marsh \& Horne 1990).

Patterson \& Raymond (1985) discussed how reprocessing of the soft X-rays generated in the boundary layer can lead to He II emission when the accretion rate exceeds $\dot{M} \gtrsim$ $1.6 \times 10^{-9} M_{\odot} \mathrm{yr}^{-1}$. This value is consistent with our results for IP Peg in the final stages of the outburst (see Sect. 4.5). Webb et al. (1999) derived an accretion rate of 


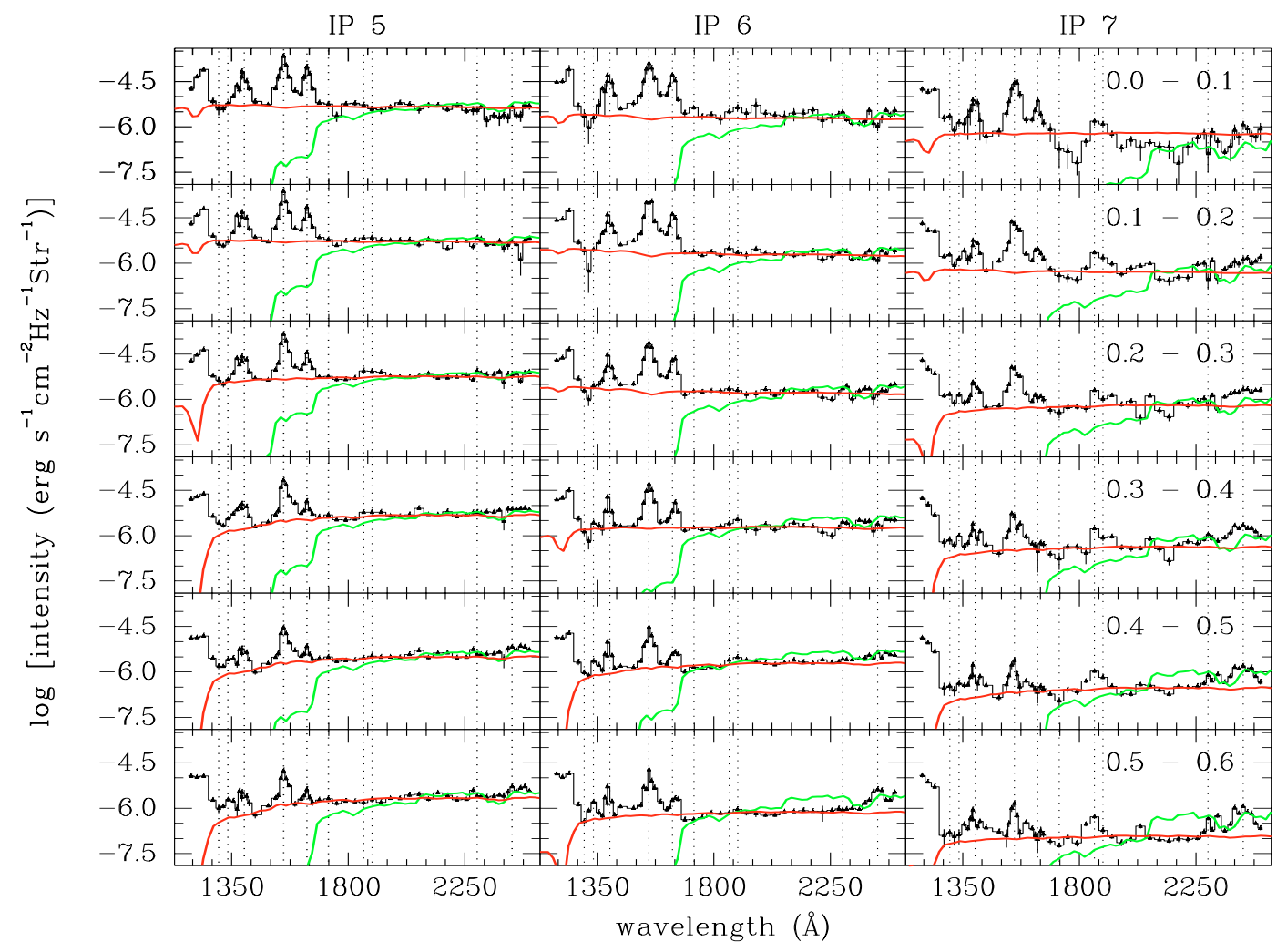

Fig. 7. Spatially resolved disc spectra $\left(90^{\circ}-270^{\circ}\right)$ for a set of six annular regions (indicated in units of $R_{L 1}$ ). The best-fit stellar atmosphere model is shown as a solid line in each panel (dark gray for the color temperature model and light gray for the brightness temperature model). Dotted lines mark the major transitions of the spectra.

$8.0 \times 10^{-9} M_{\odot} \mathrm{yr}^{-1}$ at outburst maximum, while Marsh (1988) estimated a rate of $2.2 \times 10^{-10} M_{\odot} \mathrm{yr}^{-1}$ in quiescence. The He II emission practically disappears in IP7, with only a residual emission from the innermost disc regions remaining. It is possible that the mass accretion rate in the inner disc has reduced to a level insufficient for photoionization.

Ly $\alpha$ also appears in emission at all disc radii. However, the light curve for this line is largely affected by geo-coronal emission and the results must be looked at with caution and skepticism.

For the three runs, the slope of the continuum varies in a consistent way with the distance to the disc centre. The spectra are bluer in the central parts and redder in the outer parts, indicating the existence of a radial temperature gradient in the disc.

In order to investigate the emission along the gas stream trajectory, we calculate the ratio between the spectrum of the gas stream and of the disc at same radius (Fig. 8). The results reveal that the spectrum of the gas stream is indistinguishable from the disc spectrum in the inner disc regions, but becomes clearly distinct from the disc spectrum in the intermediate and mainly in the outer disc regions. For a large range of radii $(R \gtrsim$ $\left.0.3 R_{L 1}\right)$ the continuum in the gas stream region is stronger than in the disc, in particular in IP7. In contrast to the observed in the disc spectra, where the lines appear in emission at any radius, in the ratio of the gas stream to the disc spectra the Si IV, C IV and He II lines appear in absorption in the regions where there is distinct gas stream emission in the three runs.
As discussed in Sect. 4.1, the distinct emission along the gas stream trajectory is an evidence of gas stream overflow beyond the point of impact with the disc edge.

The fact that the lines appear in absorption in the stream spectra ratio suggests the presence of matter outside of the orbital plane (e.g. chromosphere + wind) or that the disc has a non-negligible opening angle (flared disc). Horne et al. (1994) observed strong CIV emission out of the orbital plane in the dwarf nova OY Car in quiescence and suggested that this emission may arise from magnetic activity on the surface of the quiescent disc (Horne \& Saar 1991), or from resonant scattering in a vertically-extended region well above the disc. There is strong evidence of the existence of disc winds in systems with high mass accretion rates, as is observed in IP Peg in outburst (see Sect. 4.4) (Horne et al. 1994). The disc wind hypothesis was used by BHS to explain the behavior of the uneclipsed component (Sect. 4.4), while the alternative hypothesis of a flared disc is suggest from the radial run of the solid angles from the fits of model spectra (Sect. 4.5).

\subsection{The emission lines}

Figure 9 shows the radial run of the equivalent width $(E W)$ and full-width-half-maximum $(F W H M)$ of the most prominent emission lines as a function of time. The lines are stronger in the inner disc regions and decrease in strength with increasing disc radius. $\mathrm{C} I V$ is the dominant line with an $E W \simeq 400 \AA$ 


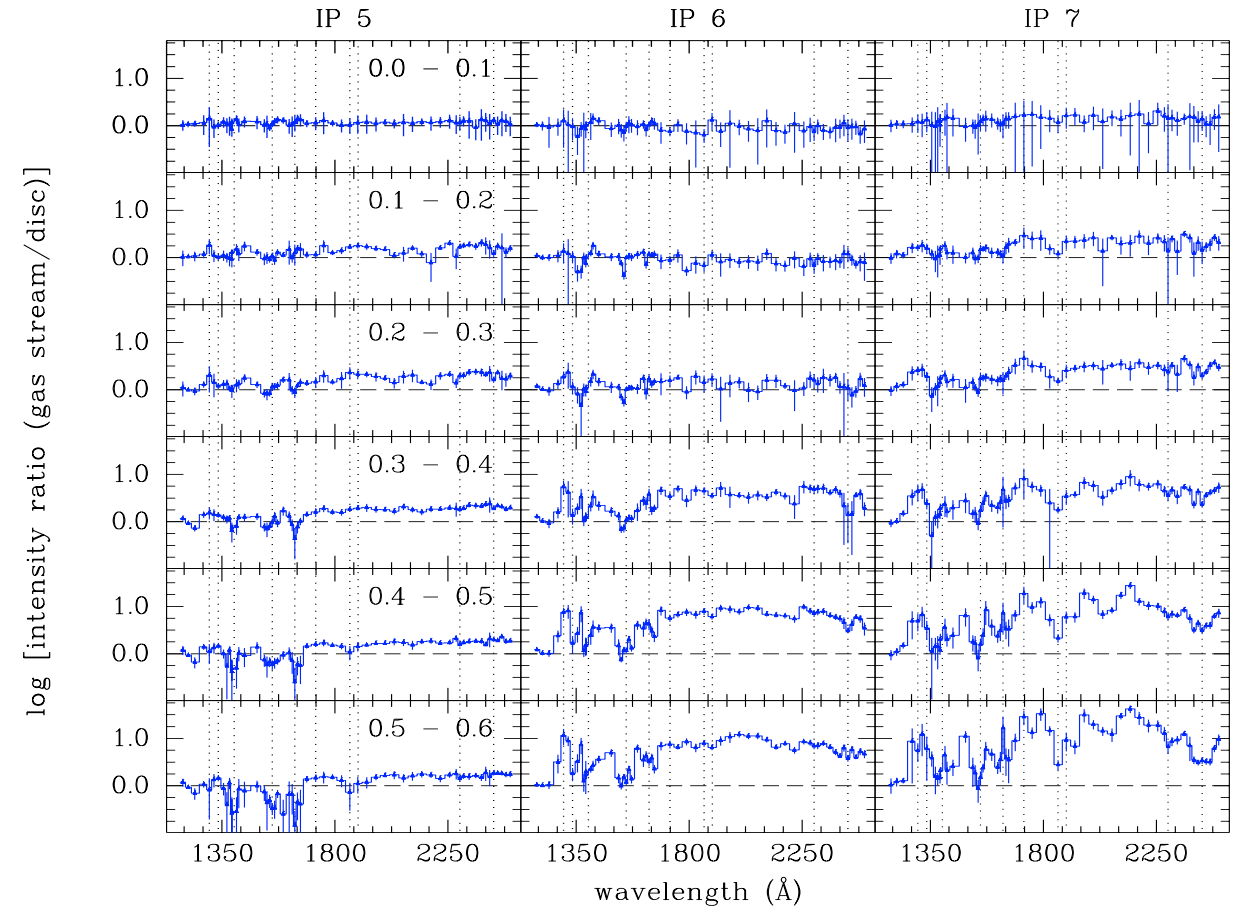

Fig. 8. The ratio between the gas stream $\left(0^{\circ}-90^{\circ}\right)$ and disc $\left(90^{\circ}-270^{\circ}\right)$ spectra as a function of radius. The notation is similar to that of Fig. 7 .

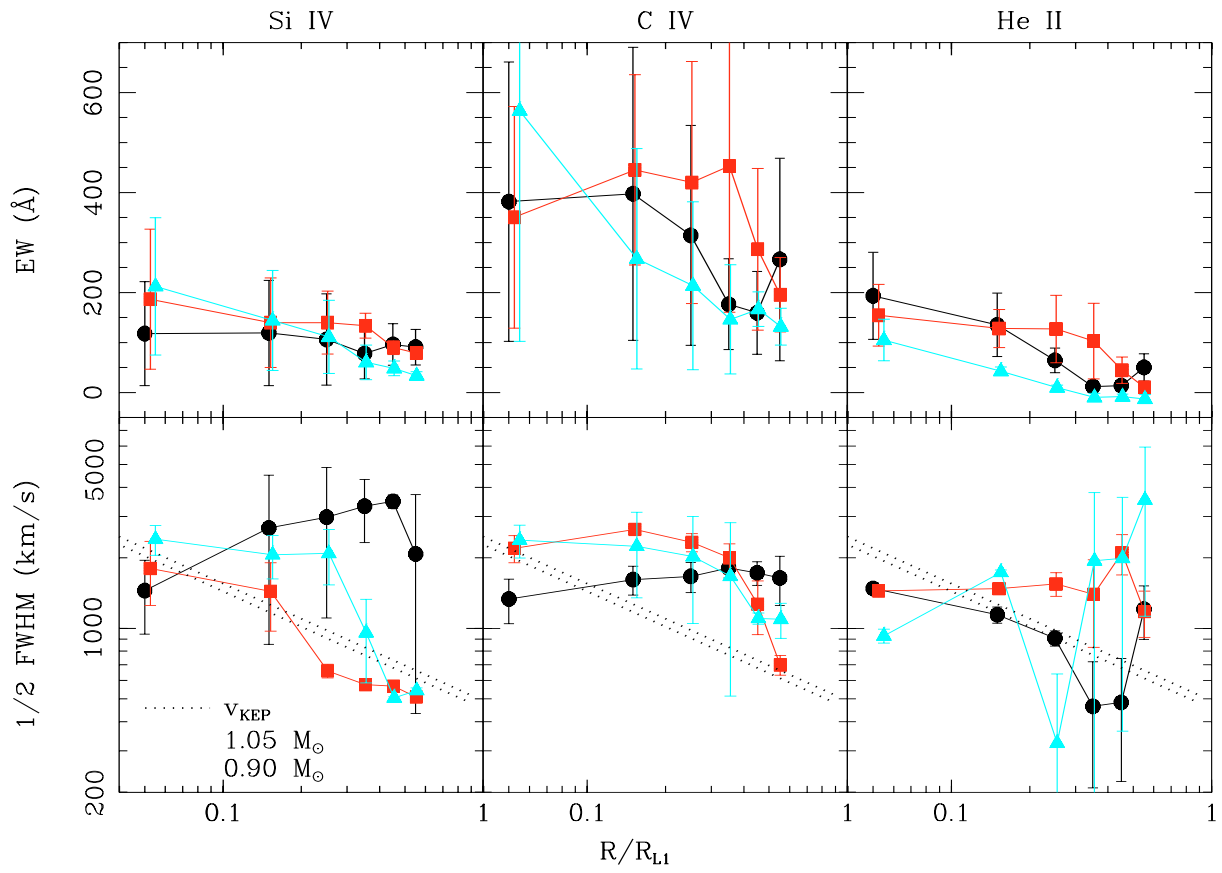

Fig. 9. Radial run of the equivalent width $(E W)$ and full width half maximum $(F W H M)$ of the most prominent emission lines as a function of time for the three runs. The run IP5 is shown as black lines with filled circles, IP6 as dark gray lines with filled squares, and IP7 as light gray lines with filled triangles. The dotted lines in the lower panels show the expected behavior for gas in Keplerian orbits around a primary star of mass $1.05 M_{\odot}$ (upper line) and $0.90 M_{\odot}$ (lower line).

at $0.1 R_{L 1}$, while $\mathrm{Si}$ IV and He II, have $E W \simeq 150 \AA$ at the same radius.

The He II emission is more concentrated in the inner regions and practically disappears in the continuum in the outer disc regions. This behavior is more pronounced in IP7. This underscores the conclusions drawn in Sects. 4.1 and 4.2 that in the last stages of the outburst the He II emission arises mostly from the disc centre. The last three points of the FWHM diagram of He II are very noisy and not reliable because the He II line is hardly discernible in the outer disc regions. In contrast, the Si IV and C IV emission is spread over the disc.

The FWHM of the C IV line is approximately constant with radius for the three runs and the derived values $(1 / 2 F W H M \simeq$ $2000 \mathrm{~km} \mathrm{~s}^{-1}$ ) are comparable to those found for others dwarf 
novae (e.g. OY Car, Horne et al. 1994). For Si IV the FWHM is approximately constant with radius in IP5, while in He II the $F W H M$ is essentially constant with radius in IP6. In the other runs (IP5 and IP7) the $F W H M$ of He II has very large error bars in the outer disc regions and the results are unreliable.

In IP5 we measure velocities of $\simeq 2000 \mathrm{~km} \mathrm{~s}^{-1}$ for C IV and $\mathrm{Si}$ IV in the outer disc regions. As a general trend, all lines show high $F W H M$ values at large radii and a flat $F W H M$ radial distribution that is markedly different from the expected behavior of gas in Keplerian orbits, where $v \propto R^{-1 / 2}$. In particular, the inferred $F W H M$ values of the outer disc regions are factor of 3-4 times larger than the Keplerian expectation. The high values of the FWHM measured in the outer disc regions indicate that the lines are not being emitted by gas in Keplerian orbits, but possibly in a chromosphere plus disc wind. The wind hypothesis was evoked in Sects. 4.1 and 4.2 to explain the absence of structures related to spiral arms and the distinct emission of the gas stream region in the line maps.

\subsection{The uneclipsed component}

The uneclipsed component was introduced in the eclipse mapping method to account for the fraction of the total light which is not coming from the accretion disc plane (e.g. light from the secondary star or from a vertically-extended disc wind) (Rutten et al. 1992).

We estimate the fractional contribution of the uneclipsed component to the total flux dividing the flux of the uneclipsed light by the average out of eclipse level at the corresponding passband. Figure 10 shows the spectrum of the uneclipsed component as well as its fractional contribution as a function of wavelength. It is dominated by strong C IV and Ly $\alpha$ emission lines (however, most of the Ly $\alpha$ emission is probably of geocoronal origin).

Previous studies in the infrared indicated that the secondary of IP Peg is a cool star of spectral-type M5 with a temperature $T \simeq 4000 \mathrm{~K}$ (Catalán et al. 2000). Therefore, one does not expect this star to contribute to the emission in the ultraviolet range. Possible irradiation effects during outburst should not affect the contribution of the secondary star to the uneclipsed light because during eclipse one sees essentially light from the side opposed to the white dwarf, which should be free from illumination by the disc and boundary layer. Moreover the facts that the lines appear in emission and that the uneclipsed component decreases toward the end of the outburst indicate that this emission is related to the outburst, discarding the hypothesis of a contribution from the secondary star.

BHT showed that the uneclipsed optical continuum contributes an increasing fraction of the total flux for longer wavelengths, reaching 10 per cent at the red end of the spectrum ( $\sim 6700 \AA)$. This suggest that the Paschen jump is in emission in the uneclipsed component. Unfortunately, our spectral coverage does not allow to test if the Balmer jump is also in emission.

The uneclipsed component of C IV accounts for 4 per cent of the total light in IP5 but decreases below the 0.5 per cent level in the other runs (Fig. 10), signaling that the vertical extension of the CIV line emitting region decreases rapidly as

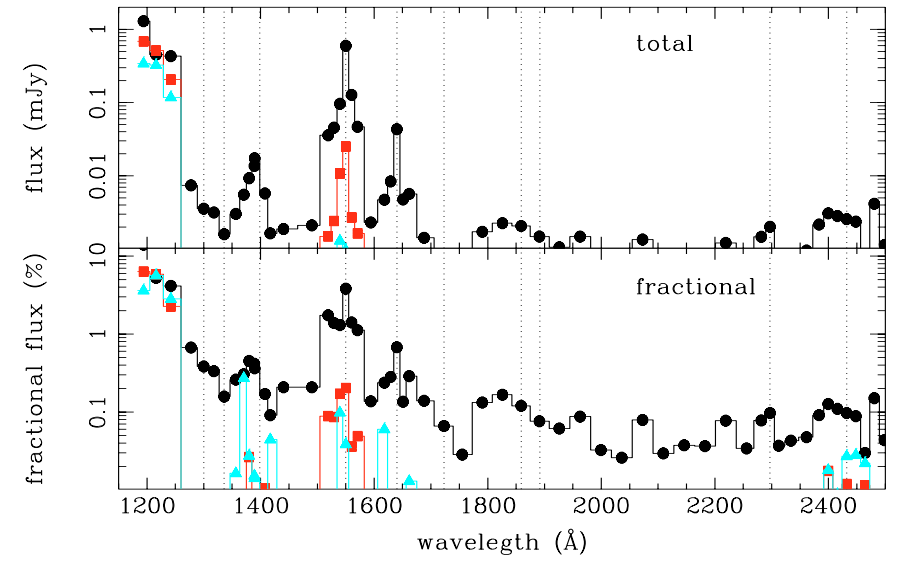

Fig. 10. Spectrum of the uneclipsed component. Top panel: total contribution. Bottom panel: fractional contribution. The notation is similar to that of Fig. 9.

the outburst approaches its end. The other lines and the continuum in the uneclipsed spectrum yield negligible fractional contributions ( $\$ 1$ per cent) to the total light at the corresponding wavelengths. For IP5, the continuum contributes $\simeq 0.1$ per cent of the total light and decreases to still smaller values at later stages ( $\$ 0.01$ per cent). The intensity of the uneclipsed component decreases much faster than that of the disc emission. If the uneclipsed component arises from a vertically-extended disc chromosphere + wind, the faster reduction of its strength in comparison with the disc emission can be interpreted as caused by the reduction of the vertical extension of this region. This corroborates the idea that the wind is associated to and powered by the outburst itself.

\subsection{The radial run of the temperature and solid angle}

The fit of model spectra to the spatially resolved continuum disc spectra allows us to infer the radial run of temperature and solid angle. It is then possible to compare the derived radial temperature distribution with the $T \propto R^{-3 / 4}$ law expected for steady-state optically thick discs, as well as to follow the evolution of this distribution in the late stages of the outburst.

We fitted blackbodies, simple LTE H I and stellar atmosphere models with metalicities $\log [M] /[H]=0.0,0.5$ and 1.0 (Kurucz 1979) to the observed spatially resolved spectra. The fits do not take into consideration possible effects of limb darkening and do not include the spectral regions containing emission lines. The statistical uncertainties in the values of the temperature and solid angle extracted from the spectral fits were estimated with a Monte Carlo procedure (Sect. 3.2).

The LTE HI fitted models resulted in high values for the column density, of order of $10^{21}$ particles $\mathrm{cm}^{-2}$, indicating that the disc gas is optically thick. The derived values of the temperature and of the solid angle are indistinguishable from those derived assuming blackbody emission.

The best-fit for blackbodies yield fits with $\chi^{2} \simeq 4-5$. These fits lead to high temperatures, of the order of $T \simeq 60000 \mathrm{~K}$ in the inner disc regions and $T \simeq 20000 \mathrm{~K}$ in the outer regions. The steady-state optically thick discs models corresponding 


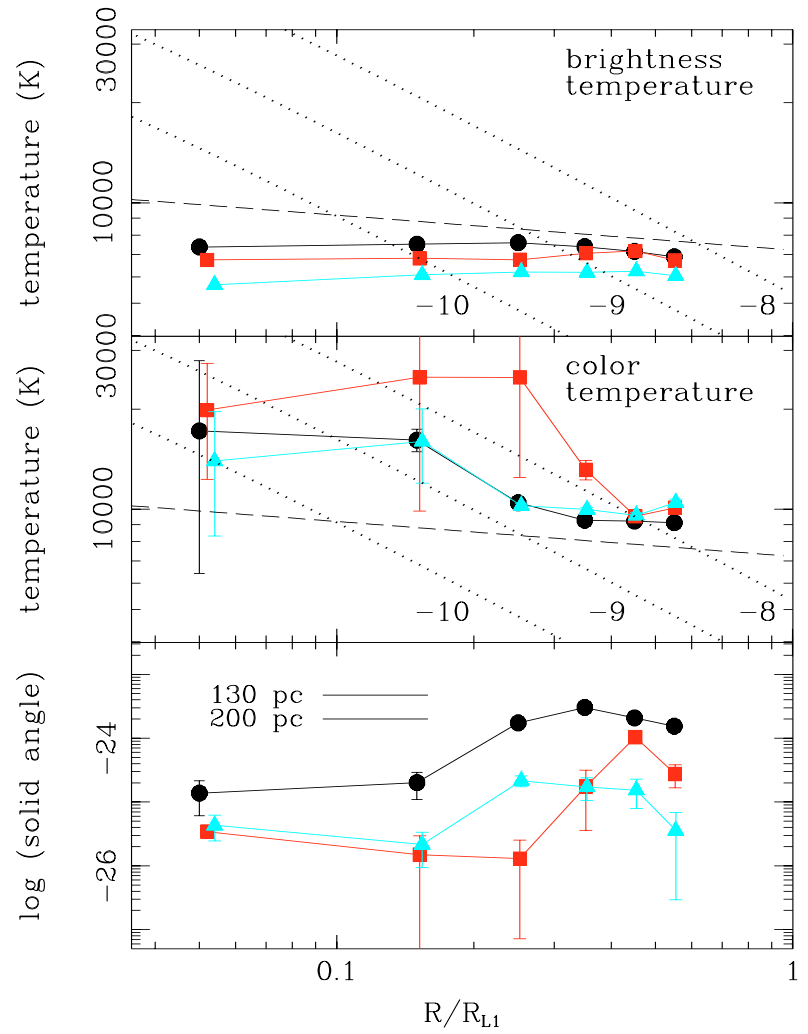

Fig. 11. Top panel: the radial run of the brightness temperature of the best-fit stellar atmosphere models to the disc spectra as a function of time for the three runs. Dotted lines show steady-state disc models for mass accretion rates of $\dot{M}=10^{-8}, 10^{-9}$ and $10^{-10} M_{\odot} \mathrm{yr}^{-1}$. The dashed line show the critical temperature above which the gas should remain in a steady, high- $\dot{M}$ regime. Middle panel: the radial run of the color temperature, the notation is the same to that of top panel. Bottom panel: the solid angle of the best-fit color temperature model in each case. Horizontal ticks mark the expected solid angle for $i=81^{\circ}$, $R_{L 1}=0.81 R_{\odot}$ and distances of $130 \mathrm{pc}$ and $200 \mathrm{pc}$. The notation is similar to that of Fig. 9 .

to these temperatures imply in mass transfer rates $(\dot{M} \gtrsim$ $10^{-7} M_{\odot} \mathrm{yr}^{-1}$ ) well above those expected for dwarf novae in outburst and even high $\dot{M}$ nova-like systems (Warner 1995).

The fits of stellar atmosphere models were performed in two different ways. First, we adopted a distance of $130 \mathrm{pc}$ to IP Peg (Martin et al. 1987). We fixed the solid angle at the corresponding value for all disc annuli and fitted the model spectra to find the brightness temperature of best-fit - i.e., the temperature of the model spectrum that produces the same intensity of the observed spectrum. The results are shown as solid light gray lines in Fig. 7. These models yield systematically poor fits to the blue side of the spectra, largely under estimating the intensities shortward of $\simeq 1800 \AA$, and result in $\chi^{2} \gtrsim 100$. The radial run of the brightness temperatures obtained from these fits are shown in the upper panel of Fig. 11. Our inferred brightness temperatures decrease monotonically from $\simeq 8000 \mathrm{~K}$ in IP5 to $\simeq 6000 \mathrm{~K}$ in IP7. The temperatures and the slope of the distribution are similar to those obtained by Bobinger et al. (1997) and by BHT, who found flat radial temperature distributions for IP Peg on the decline from outburst, with brightness temperatures respectively of 5000-9000 K and of $\simeq 5000 \mathrm{~K}$.

The inferred disc brightness temperatures are systematically higher than those of BHT although our data correspond to a later outburst stage. Together with the clear mismatch of the brightness temperature models to the short wavelengths of the observed spectra, this is an indication that (blackbody) brightness temperatures are a not a good approximation to the disc effective temperatures in this case.

Therefore, we included the solid angle as a free parameter of the model, and fitted temperature and solid angle independently for each annulus. This provides a color temperature for each annulus - we fit the shape of the spectrum and we let the solid angle account for any discrepancy in the intensity. The best-fit color temperature stellar atmosphere models are shown as solid dark gray lines in Fig. 7.

The color temperature models provide a much better fit to the data than the brightness temperature models in all cases. Among the stellar atmosphere models tested (Kurucz 1979), those of solar metalicity $(\log [M] /[H]=0.0)$ yield fits that are systematically better than those of other metalicities, resulting in $\chi^{2}$ values comparable to those obtained with blackbody models. The radial run of the derived color temperatures and the corresponding solid angles are shown, respectively, in the middle and lower panels of Fig. 11. The disc color temperature distributions are also flatter than the expected $T \propto R^{-3 / 4}$ law for steady-state optically thick discs models. However, the color temperatures are systematically higher than the brightness temperatures, with values of $T \simeq 20000 \mathrm{~K}$ in the inner disc regions and $T \simeq 9000 \mathrm{~K}$ in the outer regions.

The derived color temperatures at the end of the outburst (IP7) are everywhere higher than the critical temperature above which the gas should remain in a steady, high-mass accretion regime (Warner 1995). If the color temperatures are representative of the disc effective temperatures, this seems hard to reconcile with the disc instability model, which predicts that most of the disc gas should be back to the low viscosity, low temperature branch of the thermal limit cycle at these late outburst stages (e.g. Lin et al. 1985).

The comparatively low color temperatures obtained for the inner disc regions may be a consequence of a geometrically thick (flared) disc during outburst. If in IP Peg the inner disc regions are hidden from view by a thick disc rim, this will result in artificially low temperatures for the inner (hidden) disc regions. At the high inclination angle of IP Peg $\left(i=81^{\circ}\right)$ a moderate disc openning angle, $\alpha \simeq 10^{\circ}$, would suffice to produce this effect. Webb et al. (1999) and Morales-Rueda (2000) argued that during outbursts the disc of IP Peg is flared with an opening angle $\alpha \lesssim 15^{\circ}$.

The solid angles corresponding to the color temperature stellar atmosphere models are shown in the lower panel of Fig. 11. The solid angle increases with radius suggesting that the outbursting disc of IP Peg is possibly flared. Furthermore, the time evolution of the radial run of the solid angles suggests that the disc thickness decreases toward the end of the outburst. However, these results must be looked at with some reservations because the inferred solid angles are typically an order of 
magnitude smaller than the predicted for the expected distance to IP Peg (130-200 pc).

Because of the larger disc opening angle inferred for IP5, the inner disc regions may be hidden from view by a thick disc rim. This result can explain some apparent contradictions, p.ex. the greater inner disc temperatures found for IP6 in comparison with IP5.

\section{Conclusions}

The main results of our spectroscopic study of IP Peg during the decline of the 1993 May outburst can be summarized as follows:

1. The IP5 continuum maps show an asymmetric structure at a similar azimuth and radius as one of the spiral arms previously seen. This suggests that the spiral arms are still present in the IP Peg disc 9 days after the onset of the outburst, although the incomplete eclipse phase coverage of this run prevents a more conclusive statement. Simulations show that the incomplete phase coverage of our data does not allow us to reconstruct the other spiral arm. The other runs show no clear evidence of spiral arms.

2. The pronounced emission along the gas stream trajectory in IP6 and IP7 is an evidence of gas stream overflow.

3. Spatially resolved spectra reveal that Si IV, C IV and He II are in emission at all disc radii. The lines are stronger in the inner disc regions and decrease in strength with increasing disc radius. The FWHM of the CIV line is approximately constant with radius, in contrast to the expected $v \propto R^{-1 / 2}$ law for gas in Keplerian orbits. Similar behavior is observed in Si IV. These lines are probably emitted in a vertically-extended region (chromosphere + wind).

4. The uneclipsed component is dominated by strong C IV emission, which contributes about 4 per cent of the line flux in IP5. The fractional contribution of the uneclipsed component decreases quickly during the outburst later stages. The interpretation is that the uneclipsed component originates in a vertically-extended gas, the vertical extension of which decreases rapidly at the end of the outburst.

5. Stellar atmosphere models fitted to the spatially resolved spectra lead to color temperatures in the range $T \simeq$ $20000 \mathrm{~K}$ in the inner disc regions and $T \simeq 9000 \mathrm{~K}$ in the outer regions. For the three runs, the radial distribution of the disc color temperatures is flatter than the expected $T \propto R^{-3 / 4}$ law for steady-state optically thick discs. The derived color temperatures at the end of the outburst are everywhere higher than the critical temperature above which the gas should remain in a steady, high-mass accretion regime, in contradiction with predictions of the disc instability model for dwarf nova outburst.

6. The solid angles resulting from the spectral fit increase with disc radii. The radial run of the solid angles suggests that the disc is flared, and decreases in thickness toward the end of the outburst.

Acknowledgements. In this research we have used, and acknowledge with thanks, data from AAVSO International Data base. This work was partially supported by $\mathrm{CNPq} / \mathrm{Brazil}$ through the research grant 62.0053/01-1 - PADCT III/Milenio. R.B. acknowledges financial support from $\mathrm{CNPq} /$ Brazil through grant No. 300.354/96-7. R.K.S. acknowledges financial support from CAPES/Brazil.

\section{References}

Baptista, R. 2001, in Lecture Notes in Physics, Astro-Tomography: Imaging Methods in Observational Astrophysics, ed. H. Boffin, \& D. Steeghs (Berlin: Springer-Verlag), 307

Baptista, R., \& Steiner, J. E. 1993, A\&A, 277, 331

Baptista, R., Steiner, J. E., \& Horne, K. 1996, MNRAS, 282, 99

Baptista, R., Harlaftis, E. T., \& Steeghs, D. 2000, MNRAS, 314, 727

Baptista, R., Haswell, C. A., \& Thomas, G. 2002, MNRAS, 334, 198

Bobinger, A., Horne, K., Mantel, K., \& Wolf, S. 1997, A\&A, 327, 1023

Catalán, M. S., Smith, R. C., \& Jones, D. H. P. 2000, MNRAS, submitted

Frank, J., King, A. R., \& Raine, D. J. 1992, Accretion Power in Astrophysics - 2nd edition (Cambridge: Cambridge University Press)

Harlaftis, E. T., Baptista, R., Morales-Rueda, L., Marsh, T. R., \& Steeghs, D. 2004, A\&A, 417, 1063

Hawley, J. F., Balbus, S. A., \& Winters, W. F. 1999, ApJ, 518, 394

Horne, K. 1983, Ph.D. Thesis

Horne, K. 1985, MNRAS, 213, 129

Horne, K., \& Saar, S. H. 1991, ApJ, 374, L55

Horne, K., Marsh, T. R., Cheng, F. H., Hubeny, I., \& Lanz, T. 1994, ApJ, 426, 294

Kurucz, R. L. 1979, ApJS, 40, 1

Lin, D. N. C., Faulkner, J., \& Papaloizou, J. 1985, MNRAS, 212, 105

Marsh, T. R. 1988, MNRAS, 231, 1117

Marsh, T. R., \& Horne, K. 1988, MNRAS, 235, 269

Martin, J. S., Jones, D. H. P., \& Smith, R. C. 1987, MNRAS, 224, 1031

Martin, J. S., Jones, D. H. P., Friend, M. T., \& Smith, R. C. 1989, MNRAS, 240, 519

Morales-Rueda, L., Marsh, T. R., \& Billington, I. 2000, MNRAS, 313, 454

Patterson, J., \& Raymond, J. C. 1985, ApJ, 292, 535

Rutten, R. G. M. 1998, A\&AS, 127, 581

Rutten, R. G. M., van Paradijs, J., \& Tinbergen, J. 1992, A\&A, 254, 159

Sato, J., Sawada, K., \& Ohnishi, N. 2003, MNRAS, 342, 593

Savonije, G. J., Papaloizou, J., \& Lin, C. 1994, MNRAS, 268, 13

Sawada, K., Matsuda, T., \& Hachisu, I. 1986, MNRAS, 219, 75

Steeghs, D., Harlaftis, E. T., \& Horne, K. 1997, MNRAS, 290, L28

Stehle, R. 1999, MNRAS, 204, 687

Warner, B. 1995, Cataclysmic Variable Stars (Cambridge: Cambridge University Press)

Webb, N. A., Naylor, T., Ioannou, Z., et al. 1999, MNRAS, 310, 407

Wolf, S., Mantel, K. H., Horne, K., et al. 1993, A\&A, 273, 160

Wood, J. E., \& Crawford, C. S. 1986, MNRAS, 222, 645 
R. K. Saito et al.: HST/FOS time-resolved spectral mapping of IP Pegasi at the end of an outburst, Online Material p 1

\section{Online Material}




\section{Appendix A: Reconstruction of maps from light curves with incomplete phase coverage}

Here we address the reliability of eclipse mapping reconstructions of asymmetric brightness distributions such as spiral arms for the case of light curves with incomplete phase coverage.

First, we created eclipse maps of $51 \times 51$ pixels with two asymmetric structures at a similar position to the spiral arms observed in IP Peg by BHS. It is expected that the spiral arms become more tighty wound as the disc material cools along the decline from outburst maximum (e.g. Sawada et al. 1986; Sato et al. 2003). Because our observations of IP Peg covered the late stages of the outburst, we used configurations with four different opening angles $\phi$ for the spiral arms (e.g. Harlaftis et al. 2004), to cover a range of possible stages of spirals winding $\left(\phi=35,30,25\right.$ and $\left.20^{\circ}\right)$. The artificial spiral models are shown in Fig. A.1.

We adopt the geometry of Wood \& Crawford (1986) for IP Peg ( $i=81^{\circ}$ and $\left.q=0.5\right)$ to simulate eclipses of these brightness distributions and to generate synthetic light curves. We added Gaussian noise to the synthetic light curves to simulate real data with a signal-to-noise ratio $(S / N)$ comparable to that of the IP Peg data $(S / N=35)$.

Since the light curves of IP Peg have incomplete phase coverage, we truncated the synthetic light curves to produce the set of orbital phases of runs IP5 and IP6 (the phase coverage of IP7 is identical to IP6). Figure A.1 shows the light curves for a complete phase coverage and for the set of phases of IP5 and IP6. The shape of the eclipse changes significantly with the opening angle of the spiral arms. We applied eclipse mapping techniques to the three light curves (one with complete phase coverage, other with the phase set of IP6 and the last with the phase set of IP5) for each model and compared the results with the original maps.

Figure A.2 illustrates the eclipse geometry of IP Peg for the set of phases of runs IP5 and IP6/IP7. Arches drawn on the eclipse map connect points of constant ingress and egress phases. The pair of thick lines in each panel shows the ingress/egress arches for the first phase of the corresponding light curve $(\phi=+0.012$ for IP5 and $\phi=-0.027$ for IP6/IP7). Each pair of arches outlines a region of the disc that is being occulted by the shadow of the secondary star at a given phase. The ability of the eclipse mapping method to construct a twodimensional surface brightness map from a one-dimensional eclipse light curves stems for the fact that ingress/egress arches intersect with each other at large angles (Horne 1983).

The effect of incomplete phase coverage is to reduce the information available to reproduce structures in parts of the accretion disc. For IP6/IP7, the disc surface is fully sampled by the ingress/egress arches although about 60 per cent of the disc is only sampled by egress arches. On the other hand, about $1 / 3$ of the disc surface is not sampled by ingress or egress arches with the largely incomplete phase set of IP5. There is no information in the eclipse shape about the brightness distribution of the disc regions not sampled by the grid of arcs. Therefore, a spiral arm in the disc side approaching the secondary star (the lower hemisphere of the maps in Fig. A.2) will not affect the shape of an eclipse light curve with the phase set of IP5 and will not be recovered by the eclipse mapping method.

Figure A.1 shows the original spiral model maps, the artificial light curves generated (with complete phase coverage and with the phase sets of IP5 and IP6) and the asymmetric component of the corresponding reconstructions. The asymmetric component of each map is computed separating the radial intensity profile into radial bins of equal width. We then sort the intensities in each bin and fit a smooth spline function to the lower quartile of the intensities in each bin. The fit is then subtracted from each pixel and the resulting map is saved as the asymmetric component. This process essentially removes the baseline of the radial profile, leaving all azimuthal structure in the asymmetric map. We further set to zero all negative intensities in the asymmetric map.

The asymmetric structures in model \#1 are reasonably well reconstructed with the full phase coverage light curves (Fig. A.1). As a consequence of the intrinsic azimuthal smearing of the eclipse mapping technique (see Baptista 2001, for more details), the arms appear smeared into a "butterfly"-shape structure (Harlaftis et al. 2004). However, its position, radial and azimuthal extension are reasonably well reconstructed. Because the light curve with the set of phases of IP6 lacks most ingress phases the technique is not able to properly reconstruct the arm located in the disc region that approaches the secondary (the "blue" arm). The other spiral arm ("red" arm) is recovered in a similar fashion to the full coverage light curve. For the largely incomplete IP5 light curve, the red arm appears distorted into a crescent-shape asymmetry in the disc side moving away from the secondary star. As expected, the blue arm is not recovered in this case.

The reconstructions of model \#2 are similar to those of model \#1 in all three cases.

As the opening angle of the spirals changes, their orientation and position in the accretion disc also changes, affecting the ability of the eclipse mapping method to recover them. For model \#3, the red arm is well recovered for the IP6 light curve but is hardly discernible in the extreme case of the IP5 light curve. For model \#4, both spiral arms are well recovered with the IP6 light curve, whereas the reconstruction from the IP5 light curve starts to show hints of the blue arm.

For comparison, in Fig. A.3 we show the asymmetric component of the eclipse maps for selected continuum passbands of the IP5 and IP6 runs.

The observed asymmetries in the IP5 continuum maps are very similar to those seen in the reconstructions of models \#1 and \#2 for the same set of phases, and can be interpreted as the distorted reproduction of the "red" spiral arm from a light curve with an incomplete set of phases (missing all the eclipse ingress).

In summary, the simulations show that the eclipse mapping technique fails to recover structures in disc regions for which there is no information in the shape of the eclipse. However, asymmetric structures such as spiral arms can still be partly detected in eclipse maps derived from light curves with incomplete phase coverage such as those of the IP5 run. 
R. K. Saito et al.: HST/FOS time-resolved spectral mapping of IP Pegasi at the end of an outburst, Online Material p 3
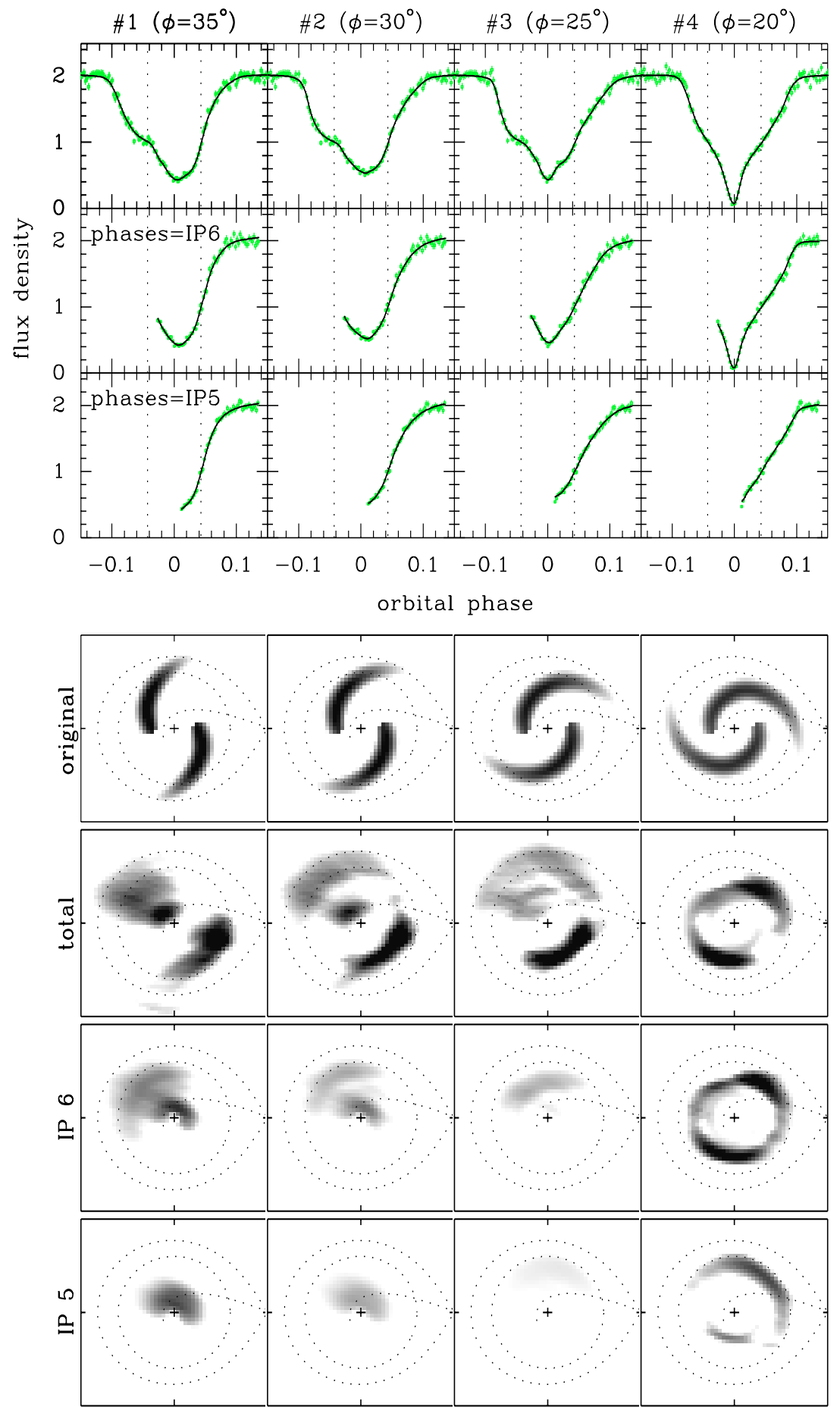

disc asymmetric components

Fig. A.1. Top panels: the synthetic light curves generated with $S / N=35$, full phase coverage and with the phase set of runs IP6 and IP5. Vertical dotted lines mark the ingress/egress times of the white dwarf. Bottom panels: the configurations of the spiral arms for four different opening angles used in the reconstructions and the eclipse maps obtained from the curves of the top panels. A cross marks the center of the disk; dotted lines show the Roche lobe, the gas stream trajectory, and a disc of radius $0.6 R_{L 1}$ (for the parameters of IP Peg); the secondary is to the right of each map and the stars rotate counterclockwise. 
R. K. Saito et al.: HST/FOS time-resolved spectral mapping of IP Pegasi at the end of an outburst, Online Material p 4

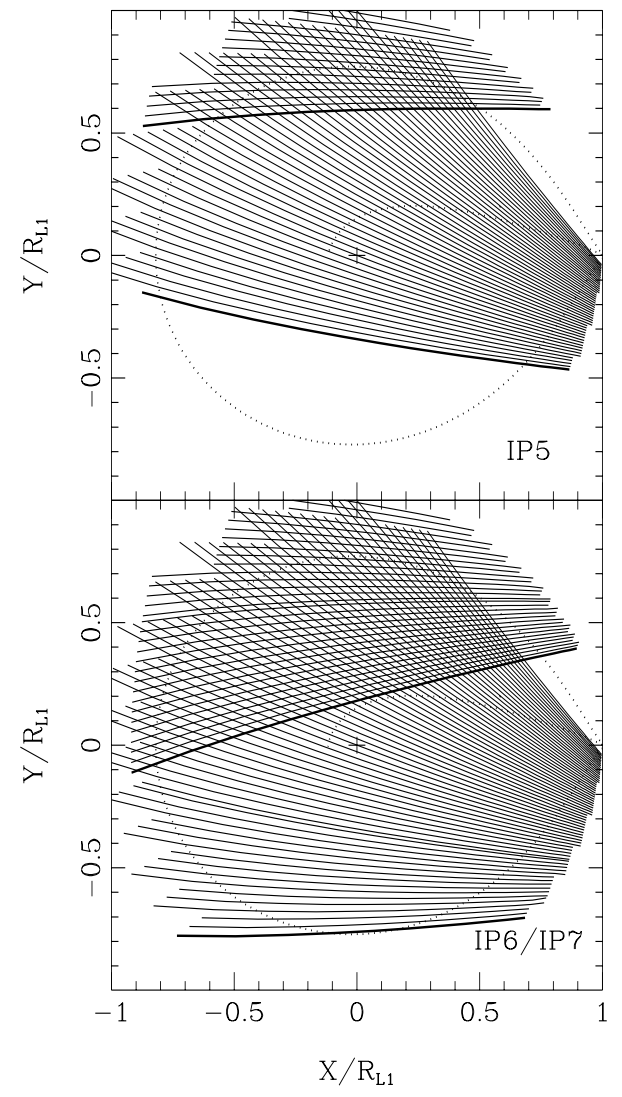

Fig. A.2. The eclipse geometry of IP Peg for the set of phases of runs IP5 and IP6/IP7. Arches (solid lines) connect points of constant ingress and egress phases. The pair of thick lines in each panel shows the ingress/egress arches for the first phase of the corresponding light curve $(\phi=+0.012$ for IP5 and $\phi=-0.027$ for IP6/IP7). Dotted lines depict the primary Roche lobe and the ballistic stream trajectory.

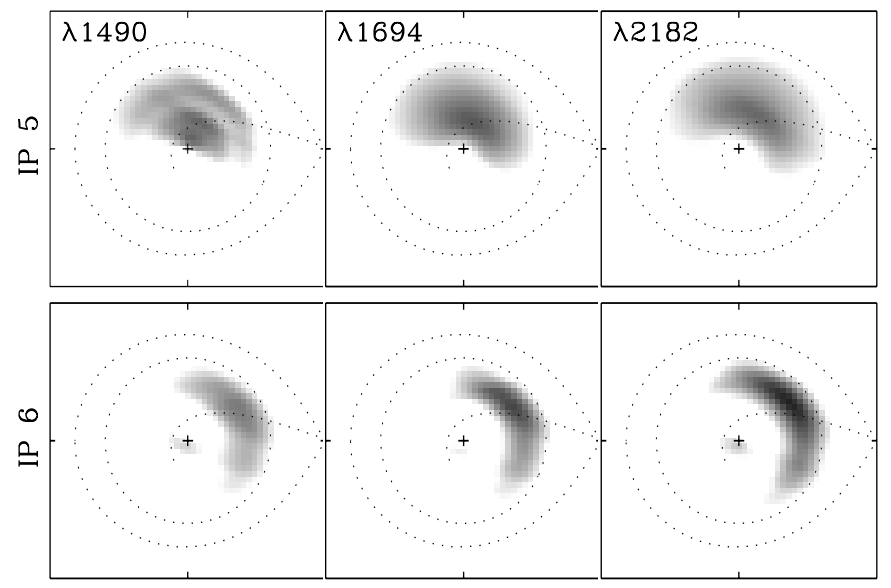

disc asymmetric components

Fig. A.3. The asymmetric component of the maps for selected continuum passbands of the runs IP5 and IP6. 